\title{
PRÁTICAS DA GESTÃO: FATORES CRÍTICOS DE SUCESSO NA GESTÃO DA INFORMAÇÃO E O PAPEL DA TECNOLOGIA DA INFORMAÇÃO NO PROCESSO DECISÓRIO DE UM HOSPITAL ${ }^{1}$
}

\section{PRACTICE MANAGEMENT: CRITICAL SUCCESS FACTORS IN INFORMATION MANAGEMENT AND THE ROLE OF INFORMATION TECHNOLOGY IN DECISION MAKING PROCESS IN A HOSPITAL}

\section{GESTIÓN PRÁCTICA: FACTORES CRÍTICOS DE ÉXITO EN GESTIÓN DE INFORMACIÓN Y EL PAPEL DE TECNOLOGÍA DE LA INFORMACIÓN EN EL PROCESO DE TOMA DE DECISIONES DE UN HOSPITAL}

Ligia Adriane Thibe

Fundação de Hematologia e Hemoterapia do Amazonas HEMOAM

ladrianethibes@gmail.com

\section{Marcia Ribeiro Maduro}

Professora da Universidade do Estado do Amazonas -UEA

Doutoranda em Administração pela Universidade Federal de Minas Gerais - UFMG/CEPEAD

marcia.maduro@gmail.com

Luis Claudio De Jesus Silva

Professor Assistente da Universidade Federal de Roraima UFRR/CADECON

Doutorando em Administração pela Universidade Federal de Minas Gerais- UFMG/CEPEAD

luisclaudiojs@gmail.com
Paulo Cesar Diniz De Araújo

Professor da Universidade do Estado do Amazonas-UEA

Doutorando em Administração pela Universidade Federal de Minas Gerais - UFMG/CEPEAD

pcdiniz1@gmail.com

Luiz Augusto De Carvalho Francisco Soares Professor da Universidade Federal do Amazonas-UFAM

Doutorando em Administração pela Universidade Federal de Minas Gerais- UFMG/CEPEAD

las10@uol.com.br

\section{Resumo}

Este artigo desenvolve uma análise sobre os fatores críticos de sucesso em uma instituição pública, tendo como base o novo paradigma da Tecnologia da Informação (TI). Seu objetivo geral é analisar a atuação da Tecnologia da Informação na organização e que papel desempenha na tomada de decisão no âmbito de um hospital do Estado do Amazonas. Neste estudo, a pesquisa foi qualitativa através da analise documental, com o objetivo de tentar identificar os fatores críticos de sucesso na gestão da informação e o papel da Tecnologia da Informação no processo decisório. O trabalho realiza a caracterização da estrutura tecnológica instalada, contextualiza a prestação de serviços à sociedade e conclui considerando os fatores e diferenciais existentes na área de tecnologia.

Palavras chave: Tecnologia da Informação; Fatores Críticos de Sucesso; Processo Decisório na Instituição Pública.

\begin{abstract}
This article provides an analysis on the critical success factors in a public institution, based on the new paradigm of Information Technology (IT). Its general purpose is to analyze the role of Information Technology in the organization and what role in decision making. In this study, the research was qualitative analysis through the Document, with the aim of trying to identify the critical success factors in information management and the role of information technology in decision making. The work done to structure characterization
\end{abstract}

\footnotetext{
${ }^{1}$ Submetido em 19 de setembro de 2013. Aceito em 02 de dezembro de 2013. O artigo foi avaliado segundo o processo de duplo anonimato além de ser avaliado pelo editor. Editores responsáveis: Márcio Augusto Gonçalves e Lucas Maia dos Santos. Reprodução parcial ou total e trabalhos derivativos permitidos com a citação apropriada da fonte.
} 
Revista de Administração Hospitalar, v.10, n.3, pp. 75-88, setembro/dezembro, 2013/ Ligia Adriane Thibe, Paulo Cesar Diniz De Araújo, Marcia Ribeiro Maduro, Luiz Augusto De Carvalho Francisco Soares, Luis Claudio De Jesus Silva

technology installed, contextualizes the provision of services to society and concludes considering the factors and differences in the area of technology.

Keywords: Information Technology; Critical Success Factors; Decision Process in Public Institution.

\section{Resumen}

En este artículo se presenta un análisis de los factores críticos de éxito en una institución pública, con base en el nuevo paradigma de la tecnología de la información (IT). Su objetivo general es analizar el desempeño de la Tecnología de la Información en la organización y el papel que en la toma de decisiones dentro de un Estado de Amazonas hospital. Este estudio fue una investigación cualitativa mediante el análisis documental, con el objetivo de tratar de identificar los factores críticos de éxito en la gestión de la información y el papel de las tecnologías de la información en la toma de decisiones. El trabajo lleva a la caracterización de la infraestructura tecnológica instalada, contextualiza la prestación de servicios a la sociedad y concluye considerando los factores y las diferencias existentes en la tecnología.

Palabras clave: Tecnología de la Información, factores críticos de éxito, la toma de decisiones en la institución pública.

\section{INTRODUÇÃO}

Este artigo tem como objetivo identificar os fatores que podem contribuir para o sucesso na prática de gestão de uma instituição pública na conjuntura da tomada de decisão com o auxílio da Tecnologia da Informação.

Tal perspectiva faz com que a gestão da informação muito discutida atualmente, estimule a criação de mecanismos de integração de dados. E, de maneira mais geral, ferramentas capazes de suportar e armazenar estas informações utilizando o conceito de realimentação. Este é, sem dúvida, o ponto de partida para o gerenciamento da informação e, não menos fundamental no processo de gestão das instituições públicas, atuando como cofator no compartilhamento de informações e, na implementação de ações para tomada de decisão.

Neste sentido, a troca de informação surgiu da necessidade do homem comunicar-se e, o advento da Tecnologia da Informação proporcionou um avanço nesta comunicação através da Internet. Surge então, um novo paradigma global, onde a evolução da tecnologia é a fronteira da relação entre o homem e o dominio da informação.

Desse modo, através das redes sociais e dos artefatos tecnológicos desenvolvidos, cada vez torna-se mais fácil superar a distância física, onde as invenções tecnológicas mais eficientes não reconhecem fronteiras e a informação pode ser utilizada como ferramenta de trabalho.

Vale ressaltar, o maior patrimônio da instituição ainda está nos recursos técnicohumano, onde cada funcionário é visto como um colaborador. Dito isso, Drucker afirma que quase todo trabalhador intelectual de uma organização terá de se tornar, ele próprio, um tomador de decisões ou de, no mínimo ser capaz de executar um papel ativo, inteligente e autônomo no processo de tomada de decisão. Além disso, a capacidade de tomar decisões eficazes determina cada vez mais à capacidade de cada trabalhador intelectual, pelo menos daqueles que estão em posições de responsabilidade, de ser eficaz, em geral. (Drucker, 2011).

Portanto, o presente trabalho pretende abordar o processo de transformação tecnológica no contexto histórico da Fundação Hospitalar Hemoam, que na percepção de alguns gestores públicos as linhas de pesquisas são importantes e direcionadas para as áreas afins. Entretanto, para outros gestores é algo que pode ser melhorado, pois se acredita que através da prática de gestão democrática no interior da tecnologia, poderá se eliminar o individualismo, a acomodação e indisponibilidade em que se reduz o conhecimento da Tecnologia da Informação. 


\section{UMA VISÃO GERAL DO PROCESSO}

Visando capturar aspectos do processo decisório baseado no desenvolvimento da tecnologia da informação, apresentamos um caso prático: uma instituição pública (Fundação Hospitalar Hemoam) cujos processos informatizados de sistemas técnicos e administrativos num primeiro momento, alcançavam os objetivos e necessidades da organização.

Para dar os primeiros passos nessa direção, devemos levar a tecnologia a sério, utilizando-a como ponto de partida desta investigação; precisamos localizar o processo de transformação tecnológica revolucionária, ou seja, ser informacional como atributo de uma organização social, focada no processamento e uso da informação através das condições permitidas pelas novas tecnologias no contexto social da organização em que ele ocorre e pelo qual está sendo moldada; ser capaz de buscar essa identificação no contexto da transformação tornar-se tão poderosa quanto a transformação econômica e tecnológica no registro da nova história (Castells, 1999).

Contudo, para Prahalad se não há capacidade para a eliminação dos gargalos, que impedem ou limitam os recursos, se a empresa não aprendeu a fazer mais com menos e, em outras palavras, os riscos de ser "estratégica" são totalmente proporcionais ou mais do que proporcionais às possíveis recompensas, não há vantagem em ser estratégico. Quanto maiores são os investimentos, maiores os retornos, mas também pode ser maior o número de problemas causados, ou seja, podem gerar desastres maiores. (Prahalad, 2005).

Conforme se observa a colocação dos autores podemos dizer que, a princípio a tecnologia não determinou o crescimento da organização, apenas acompanhou sua transformação, uma vez que os processos principais não foram afetados pela carência tecnológica. No entanto, concluímos ao analisarmos a instituição como um processo social; o cenário é redesenhado em processos onde a informação e o conhecimento permitem que os gestores tomem as decisões, que são atos planejados, mas para se tornarem ações executáveis exigem inovações na estrutura tecnológica para suportar a carga destas informações; a complexa gama de atividades empreendidas e representar suas atividades.

\subsection{A MUDANÇA NO PARADIGMA DA TECNOLOGIA E O IMPACTO HISTÓRICO E SOCIAL}

As novas tecnologias consolidadas nos anos 70, 80 e 90, especialmente na área de microeletrônica, colocaram o ser humano no centro de uma transformação por muitos comparada àquela promovida pela Revolução Industrial no final do século XVIII. (Coleção Prêmio Luís Eduardo Magalhães, 2004).

Para Castells, o fator histórico mais decisivo para a aceleração, encaminhamento e formação do paradigma da tecnologia da informação e para a indução de suas conseqüentes formas sociais foi/é o processo de reestruturação capitalista, empreendido desde os anos 80 , de modo que o novo sistema econômico e tecnológico pode ser adequadamente caracterizado como capitalismo informacional. Onde as organizações alheias aos adventos da nova era da tecnologia não conseguiriam ter perspectivas para manter suas atividades em um mundo globalizado e interconectado. A decisão estava entre tornar-se global e informacional ou, então, sucumbir. (Castells, 1999) 
Revista de Administração Hospitalar, v.10, n.3, pp. 75-88, setembro/dezembro, 2013/ Ligia Adriane Thibe, Paulo Cesar Diniz De Araújo, Marcia Ribeiro Maduro, Luiz Augusto De Carvalho Francisco Soares, Luis Claudio De Jesus Silva

Entender a complexidade e relacionar os vários ramos do conhecimento é uma ansiedade humana antiga e, sua base está na reciprocidade e integração da informação, que com o advento tecnologia tornou-se possível. E, para que a informação seja histórica e atual, é necessária sua realimentação.

Neste sentido, de acordo com Chiavenato a Cibernética é uma teoria dos sistemas de controle e baseada na comunicação (transferência de informação) entre o sistema e o meio e dentro do sistema e do controle (retroação) da função dos sistemas com respeito ao ambiente. (Chiavenato, 2003).

Assim, os computadores criados para reproduzir o comportamento humano em sua total complexidade, inicialmente de cunho apenas cientifico, têm agora foco voltado para sociedade em geral. Sendo utilizado em grande escala nas redes sociais com a finalidade de comunicação direta entre indivíduos, mas também como recurso para compor as atividades das organizações e gerenciar o conhecimento dos serviços.

É nesse sentido que a tecnologia por não reconhecer fronteiras, revolucionou o mundo todo com a capacidade de superar as limitações físicas, a exemplo do correio eletrônico e a grande capacidade de armazenamento de dados. Neste contexto, o mundo digital introduz um novo comportamento nas relações sociais, criando uma forte influência no cotidiano, redefinindo características da condição humana.

Para Castells a construção de uma nova cultura baseada na comunicação multimodal e no processamento digital de informações cria um hiato geracional entre aqueles que nasceram antes da Era da Internet (1969) e aqueles que cresceram em mundo digital. A emergência de um novo paradigma tecnológico organizado em torno de novas tecnologias da informação, mais flexíveis e poderosas, possibilita que a própria informação se torne o produto do processo produtivo. Para a informação outrora utilizada como fonte de dados, no novo paradigma informacional tornar-se o próprio produto, definindo uma linha entre ser dono do poder propiciado pela informação ou estar em uma estrutura aquém da que as organizações robustas dispõem. Portanto, afirmar que a produtividade gera crescimento econômico e que ela é uma função da transformação tecnológica equivale a dizer que as características da sociedade são os fatores cruciais subjacentes ao crescimento econômico, por seu impacto na inovação tecnológica. (Castells, 1999).

Podemos então dizer que tecnologia e informação guardam entre si uma estreita articulação, sendo que ambas são fundamentais para o subsidio das atividades atuais e desenvolvimento nas grandes metrópoles. O homem torna-se cada vez mais dependente das inovações tecnológicas, as atividades e rotinas que ganharam significativa otimização nos processos e recursos, permitiram aos indivíduos o ganho do tempo. Assim, acirrou-se uma busca incontrolável pelos recursos fornecidos por inovações tecnológicas, redefinindo a interação entre a raça humana, onde passa a existir o isolamento e a mudança do comportamento em função da tecnologia.

Sobre isso: estabelecem-se (...) novas hierarquias geopolíticas, definidas com base em novos diferenciais socioespaciais, refletindo fundamentalmente desiguais disponibilidades de informações e conhecimentos estratégicos, bem como desiguais posições no âmbito dos fluxos e dos fixos que compõem as redes de informações e comunicação em escala planetária. (Coleção Prêmio Luís Eduardo Magalhães, 2004).

\subsection{A TECNOLOGIA DA INFORMAÇÃO NA INSTITUÇÃO PÚBLICA BRASILEIRA}

A Era do Computador predominou até o final da década de 70 e a Era da Informação começou a partir dos primeiros anos da década de 80, sendo que muitas empresas ainda estão na transição entre as duas e que no Brasil esta mudança aconteceu por volta da metade da década de 80. Na Era do Computador a competência necessária era técnica e o estilo gerencial 
era o de controle, no entanto na era da informação há o envolvimento em nível de alta gerência; a capacidade dos gestores de TI precisa estar no nível conceitual para abranger os recursos disponíveis da Era da Informação; o planejamento é estratégico e o estilo gerencia é o da influência. (Albertin, 2002).

Portanto, a tecnologia redefiniu o processo de comunicação global a partir da década de 80, através de inovações tecnológicas, que desde então estão se aprimorando e, sob esta perspectiva determina-se um novo comportamento social e profissional diferente do que se conhecia até então.

A função principal da Tecnologia da Informação (TI) é alavancar serviços, manter os recursos tecnológicos utilizados em funcionamento e garantir a continuidade das atividades dos serviços disponibilizados. No entanto, tal propósito exige que a gestão das atividades de TI esteja de acordo com a estratégia da instituição.

Apesar dos muitos recursos da TI a falta de alinhamento à torna de difícil entendimento e, popularmente citada como "caixa preta", a TI tem em seu interior um tipo de comunicação impar em relação à organização e uma forma de ação diferenciada e não compreendida, o que na maioria das organizações traz as atividades de TI grandes embaraços no apoio as inovações.

Cabe ressaltar, que muitos dos serviços de TI têm soluções terceirizadas cujo controle da continuidade esta estrategicamente sob terceiros e, uma grande quantidade de recursos empregados. Mas sem dúvida, a maior lacuna da área de TI esta na falta do alinhamento da área de TI com a estratégia da organização, tornando-a isolada e, onde as decisões tomadas lhe são alheias, criando uma ilha de conhecimento sem interatividade com a gestão.

Nas organizações públicas a TI precisa estar integrada, visualizando o objetivo estratégico da organização e dos serviços prestados por ela. E, acima de tudo consolidada em um sistema que possa ser operado de forma eficiente fornecendo informações para um efetivo controle da gestão. Dessa forma, garantir que os serviços controlados pela TI estejam disponíveis quando requeridos.

Neste contexto, é necessário questionar quais são as prioridades de investimento? Quais tecnologias e software são mais adequados para a instituição? Como será disponibilizada a infraestrutura necessária para o funcionamento da tecnologia adquirida? Qual a necessidade de investimento nos profissionais de TI para que estejam confortáveis e mais produtivos?

A TI diferente do que muitos imaginam, vai além de serviços de compra e manutenção de equipamentos ou suporte a sistemas, pois seu melhor esforço deve ser empregado em gerenciar informações e recursos, cujo foco é planejar e implementar soluções para a gestão estratégica da instituição, garantindo que seus objetivos sejam alcançados.

Além disso, a tecnologia tem como premissa prover o acesso às informações de forma rápida e confiável auxiliando na tomada de decisão. Cabe ressaltar, a tecnologia muda constantemente e para que se construa a gestão da informação eficiente é importante a adequação da TI neste processo.

Então para o gerenciamento eficiente da TI e a autonomia das informações é preciso a utilização do conceito de feedback onde concordamos com Chiavenato quando afirma: um servomecanismo de precisão capaz de se auto-corrigir rapidamente e ajustar-se a um alvo em movimento variável. (Chiavenato, 2003). Além do conceito de sinergia de informações onde Rezende diz que: o conceito genérico de sinergia é coerência, integração, relação horizontal e vertical. Também pode ser vista como a multiplicação da combinação de recursos, planos com o mesmo objetivo e direção. Os recursos e as informações utilizadas conjuntamente produzem um efeito maior. (Rezende, 2011) 
Revista de Administração Hospitalar, v.10, n.3, pp. 75-88, setembro/dezembro, 2013/ Ligia Adriane Thibe, Paulo Cesar Diniz De Araújo, Marcia Ribeiro Maduro, Luiz Augusto De Carvalho Francisco Soares, Luis Claudio De Jesus Silva

Dessa forma, é imprescindível que a TI seja reconhecida como área estratégica na instituição. Daí precisa reorientar-se na prestação dos serviços e concluir os objetivos propostos, satisfazendo os requisitos de negócios da organização.

\subsection{FATORES CRÍTICOS DE SUCESSO COMO ESTRATÉGIA ORGANIZACIONAL}

Chegar ao futuro primeiro é mais uma função da capacidade de dinamizar os recursos do que dos recursos em si. A capacidade de dinamização dos recursos deriva-se não de uma arquitetura estratégica elegante, mas de uma noção profunda de propósito, um sonho amplamente compartilhado, uma visão realmente sedutora da oportunidade que o amanhã oferece. (Prahalad, 2005).

A partir do cenário delineado, a seguir, apresenta-se um relato da evolução da Fundação de Hematologia e Hemoterapia do Amazonas (FHEMOAM) em um contexto histórico: a FHEMOAM iniciou suas atividades na hematologia e hemoterapia no ano 1982 nas dependências do Hospital Universitário Getúlio Vargas, órgão vinculado a Universidade Federal do Amazonas. Em 1986, obteve através da criação do Programa Nacional do Sangue e Hemoderivados, uma nova perspectiva de futuro tornando-se hemocentro integrante da rede nacional, utilizando a partir daí a informatização do sistema de acompanhamento à doação de sangue para o armazenamento das informações do processo. No entanto, a partir de 1989 ao ser transformado em fundação de direito privado passou a desenvolver serviços de análises clinicas e ensino e pesquisa, e a desempenhar suas atividades de hemoterapia no interior do Estado.

Seguindo sua trajetória inovadora com um ponto de vista futurista a instituição transforma-se em 2011 na Fundação Hospitalar de Hematologia e Hemoterapia do Amazonas, com o planejando de construção do hospital a partir de 2012 e que irá atender a demanda dos casos que exigem um ambiente hospitalar. E ainda vai além, prevendo a construção do Banco de Sangue do Cordão Umbilical e Placentário e o Banco de Sangue Raros.

Neste sentido, visando maior segurança e qualidade do sangue, a FHEMOAM implanta através do Ministério da Saúde um sistema que utiliza a quantificação de carga viral do HIV e HCV para detecção de ácido nucléico de HIV e HCV em bolsas de sangue. Novamente renovando, é agora um projeto piloto de implantação da hemorrede brasileira, que torna o Amazonas o hemocentro referência na região norte; tem sob sua responsabilidade as rotinas de HIV e HCV da nova plataforma do laboratório de biologia molecular, atendendo ainda os estados de Roraima, Acre e Rondônia.

Dito isso, concluímos que muito se fez com honestidade e transparência nesta organização governamental. No entanto, a excelência prestada pelos serviços da Fundação Hospitalar Hemoam não impediu que outro banco de sangue viesse a se estabelecer nesta capital; o Instituto de Hemoterapia do Amazonas (IHAM) inicia assim suas atividades em 2003, concorrendo com o FHEMOAM no abastecimento de sangue para os hospitais privados.

Porém em detrimento de inadequações verificadas no laboratório de sorologia, que comprometiam a qualidade do sangue coletado e distribuido pelo IHAM, a Agência Nacional de Vigilância Sanitária (ANVISA) e a Fundação de Vigilância em Saúde do Estado (FVS), determinaram a interdição temporária do IHAM.

Por mais rápida que tenha sido a permanência do concorrente, e a vasta experiência da FHEMOAM na hemoterapia, a instituição tem agora uma dolorosa lição que requer sua atenção, que é um estudo profundo para alavancar seu portfólio de recursos dos produtos hemoderivados do sangue e técnico-humano especializado para o desempenho das atividades em expansão. 
Revista de Administração Hospitalar, v.10, n.3, pp. 75-88, setembro/dezembro, 2013/ Ligia Adriane Thibe, Paulo Cesar Diniz De Araújo, Marcia Ribeiro Maduro, Luiz Augusto De Carvalho Francisco Soares, Luis Claudio De Jesus Silva

\section{METODOLOGIA}

Neste estudo utilizou-se a Pesquisa Qualitativa através da analise Documental, com o objetivo de tentar identificar os fatores críticos de sucesso na gestão da informação e o papel da Tecnologia da Informação no processo decisório. A pesquisa Documental foi realizada objetivando consulta a legislação, a fim de completar as informações obtidas por documentos, como também pode se constituir numa técnica valiosa de abordagem de dados qualitativos, seja completando as informações obtidas por outras técnicas, seja desvelando aspectos novos de um tema ou problema (André, 1986, p.38). A investigação Documental é a realizada em documentos conservados no interior dos órgãos públicos e privados de qualquer natureza, ou com pessoas. (Vergara, 2011).

No primeiro momento foi realizado um estudo bibliográfico sobre autores que trabalham a temática; documental sobre o tema central do estudo de caso. Nesta primeira etapa, selecionaram-se os autores mais adequados para o estudo sobre a era da informação e as decisões gerenciais. Em seguida, procedeu-se a seleção de Documentos, tais como: Regimento Interno 2010, Procedimento Operacional Padrão 2011, Relatório de Atividades do Departamento de Sistemas 2011 e Sistemas em uso na instituição, que falam sobre as atividades desenvolvidas na instituição e pela Tecnologia da Informação, importantes para o esclarecimento da pesquisa.

$\mathrm{Na}$ analise dos dados buscou-se entender o papel exercido pela Tecnologia da Informação na instituição, particularmente na gestão da informação e o apoio no processo decisório organizacional.

A última fase do artigo apresenta uma apreciação das fontes documentais, especialmente no que diz respeito às atividades prioritárias da instituição e a disponibilidade da tecnologia da informação no processo, procurando explicitar as atividades relacionadas entre as áreas fins e a tecnologia.

Portanto, pretende-se com este artigo apresentar uma análise de documentos importantes de uma instituição pública e demonstrar os pontos críticos de sucesso, traçando considerações e proposições de melhorias relacionadas ao uso da Tecnologia da Informação.

4 ANALISANDO UM CASO PRÁTICO DE UMA INSTITUIÇÃO PÚBLICA: “do uso da Tecnologia da Informação à Gestão da Informação na FHEMOAM e os fatores críticos de sucesso"

O cenário atual da instituição requer que seja demandado um olhar plural nos processos associados à área de Tecnologia da Informação. Essas mudanças precisam ser inseridas em um processo participativo, onde o profissional de TI possa ser co-decisor. Para tal, é necessário que seja aplicado um esforço coletivo na reestruturação dentro da TI, enquanto área critica com contribuição significativa e determinante para o sucesso das atividades fins, cuja premissa é a qualidade nos serviços prestados a população.

Para O'Brien a sabedoria convencional diz que o conhecimento é poder, mas caso ele seja colhido desordenadamente, pode tornar-se menos poderoso. Na era informacional não é diferente, é preciso saber colher os dados para tornar-los informações produtivas, isto é, a classe emergente de aplicações concentra-se no apoio personalizado a decisões, modelagem, recuperação de informações, armazenamento de dados, cenários hipotéticos e relatórios. (O’Brien, 2010).

É extremamente importante um diagnóstico e a tratativa nos processos de TI desta instituição, pois a área de TI tem o papel de comportar as atividades desenvolvidas nas diversas áreas da organização e armazenar em sistemas, além de prover a infraestrutura para o funcionamento destes. E para que a informação torne-se domínio do conhecimento é preciso 
transcender as barreiras de comunicação e estabelecer indicadores desejados pelas atividades; definir a partir daí os indicadores de TI, de forma que venha a controlar os serviços da instituição.

Um ponto vital para o desempenho satisfatório no uso das tecnologias refere-se ao usuário que requer melhor treinamento. Nenhuma tecnologia é eficaz se não for utilizada adequadamente, ou seja, se seu uso é incompleto por falta de habilidade; é preciso a percepção desta necessidade em coordenar programas de treinamento e a renovação deste conhecimento.

Outrossim, é fundamental a compreensão por parte dos colaboradores, sobre a importância do papel que executam no contexto da instituição e social; incluindo os da TI que na sua grande maioria não tem vinculo efetivo com a instituição, e que com a rotatividade de pessoal acarreta a perda da memória do conhecimento. Dessa forma, as atividades desenvolvidas serão melhores desempenhadas e não mais existirão processos isolados ou atividades diferenciadas; apenas o bem comum à sociedade, objetivo principal da organização.

A Tecnologia da Informação e seus recursos nem sempre resolvem os problemas nas empresas e muito menos as organizam. É preciso para efetivar esta organização a gestão da informação, ou seja, o gerenciamento dos recursos e tecnologias disponíveis. Tecnologia por tecnologia, sem planejamento, sem gestão e ação efetiva, não traz contribuição para a empresa. (Rezende, 2011).

De fato, a tecnologia da informação é um pilar nas grandes organizações, contribuindo efetivamente nas atividades desenvolvidas e processos desempenhados nas rotinas. Portanto, precisa-se na FHEMOAM enquanto organização definir quais metas deverão ser atingidas com a tecnologia da informação e quais os indicadores das metas? Com base nestes indicadores incluir no modelo da organização as funções que compreende o papel da TI.

Diante desse quadro, a perspectiva de inserir novas tecnologias de gerenciamento dos processos e do dominio da informação institucional é cada vez mais evidente. Mas para que isso aconteça, além dos indicadores é preciso que se tenha uma visão geral da estrutura implantada na TI; e assim partir para a adoção de ferramentas de gerenciamento para dirigir e controlar a estrutura tecnológica e atividades envolvidas nos processos da organização.

4.1 AS ATIVIDADES DESENVOLVIDAS NA FHEMOAM: "percepção da importância na sociedade"

As atividades desempenhadas pela FHEMOAM são relevantes no contexto da assistência à saúde no estado do Amazonas, considerando o trabalho desenvolvido de alta especialização. Assim, a FHEMOAM presta serviço à sociedade disponibilizando a população serviços nas áreas:

\section{De Hematologia:}

- Atendimento Ambulatorial e Hospitalar a pacientes portadores de doenças hematológicas;

- Atendimento de curto prazo para tratamento de Quimioterapia e Transfusão Sanguínea;

2. De Hemoterapia:

- Exames para fins de Diagnósticos de doença no sangue;

- Recrutamento; Triagem; Coleta de sangue;

- Produção e Distribuição de sangue e hemocomponentes;

- Atendimento ambulatorial de doadores de sangue inaptos;

\section{De Laboratório:}


Revista de Administração Hospitalar, v.10, n.3, pp. 75-88, setembro/dezembro, 2013/ Ligia Adriane Thibe, Paulo Cesar Diniz De Araújo, Marcia Ribeiro Maduro, Luiz Augusto De Carvalho Francisco Soares, Luis Claudio De Jesus Silva

O Exames para acompanhamento de mães RH negativo, teste do pezinho, exclusão de paternidade;

- Para liberação da doação de sangue: Laboratório de Sorologia, Laboratório de Imunohematologia e Laboratório de Pesquisa de Anticorpos antieritrocitários;

○ Para geração dos hemocomponentes: Laboratório de Fracionamento do Sangue;

- Para distribuição de sangue para estoque nos hospitais e transfusão de paciente: Laboratório de Compatibilidade e Distribuição de Sangue;

- Atendimento da população geral: Laboratório de Bioquímica, Laboratório de Imunologia, Laboratório de Hormônios, Laboratório de Hematologia, Laboratório de Hemostasia, Laboratório de Imunofenotipagem e Hemoglobinas, Laboratório de Biologia Molecular, Laboratório de Urinálise e Parasitologia;

\section{De Ensino e Pesquisa}

- Capacitação de pessoal para desempenho das atividades de Hematologia e Hemoterapia;

- Treinamento técnico em Hemoterapia;

- Palestras, Seminários e Cursos;

- Participação em vídeos conferências da Rede Universitária de Telemedicina;

- Residência médica em Hematologia e Hemoterapia;

○ Mestrado em: Hematologia;

- Desenvolvimento de pesquisas para testes diagnósticos de doenças no sangue como: Hepatite B, Leucemias, Malária e Doenças Transmissíveis pelo Sangue;

- Estágio curricular para graduação em Medicina, Enfermagem, Farmácia, Bioquímica, Psicologia, Biomedicina, Serviço Social e área afins;

Os dados do doador e pacientes envolvem o caráter sigiloso e, apesar da base de dados não ser de grande porte seu teor torna significativa sua segurança, no qual é tal utilizado um sistema fechado para armazenamento dos dados.

\begin{tabular}{l|lllllllllllll}
\hline \multicolumn{10}{c}{ Tabela 1 - Doações Coletadas no Período de 2011 e 2012 } \\
\hline & \multicolumn{10}{c}{ Meses } \\
\hline \multirow{3}{*}{2011} & Jan & Fev & Mar & Abr & Maio & Jun & Jul & Ago & Set & Out & Nov & Dez \\
& & & & & & & & & & & & & \\
2012 & 4.080 & 4.510 & 3.779 & 3.792 & 4.084 & 4.154 & 3.723 & 3.761 & 3.627 & 3.206 & 4.507 & 3.516 \\
& & & & & & & & & & & & & \\
\hline
\end{tabular}

Pela Tabela 1 pode-se observar que com exceção dos meses de Janeiro, Fevereiro e Junho o quantitativo de doações teve relativo acréscimo no ano de 2012 , não podendo ser comparados os meses de Novembro e Dezembro de 2012.

\begin{tabular}{c|ccccccccccccc}
\hline \multicolumn{10}{c}{ Tabela 2 } & Exames de Sorologia Básica para Doações de Sangue no Período de 2011 e 2012 \\
\hline & \multicolumn{10}{c}{ Meses } \\
& Jan & Fev & Mar & Abr & Mai & Jun & Jul & Ago & Set & Out & Nov & Dez \\
\hline
\end{tabular}


Revista de Administração Hospitalar, v.10, n.3, pp. 75-88, setembro/dezembro, 2013/ Ligia Adriane Thibe, Paulo Cesar Diniz De Araújo, Marcia Ribeiro Maduro, Luiz Augusto De Carvalho Francisco Soares, Luis Claudio De Jesus Silva

\begin{tabular}{l|lllllllllllll}
\hline 2011 & 31.544 & 36.293 & 31.430 & 30.871 & 33.101 & 33.154 & 30.853 & 30.484 & 31.066 & 25.646 & 35.300 & 30.831 \\
& & & & & & & & & & & & & \\
2012 & 26.944 & 32.699 & 34.854 & 31.477 & 37.929 & 29.754 & 30.894 & 35.571 & 27.000 & 32.089 & - & - \\
\hline \multicolumn{10}{c}{ Fonte: Elaborado pelos autores }
\end{tabular}

A cobertura de exames de sorologia básica para as doações de sangue coletadas é demonstrada na Tabela 2, onde pode ser observado que em 2011 foram realizados o total de 380.573 exames e em 2012 ainda restando dois meses para conclusão do ano já foram realizados 319.211, pois novos exames mais sensíveis foram inseridos na rotina e têm sido utilizados na execução dos testes sorológicos aumentando a qualidade e a segurança transfusional.

Tabela 3 - Agendamento Médico de Pacientes para o ano de 2012

\begin{tabular}{|c|c|c|c|}
\hline \multicolumn{2}{|c|}{ Agendamento $1^{\circ}$ Semestre } & \multicolumn{2}{|c|}{ Agendamento $2^{\circ}$ Semestre } \\
\hline Jan & 2.285 & Jul & 3.716 \\
\hline $\mathrm{Fev}$ & 1.896 & Ago & 3.769 \\
\hline Mar & 3.299 & Set & 2.658 \\
\hline Abr & 2.856 & Out & 3.478 \\
\hline Mai & 3.491 & Nov & 890 \\
\hline Jun & 3.327 & Dez & 317 \\
\hline l Parcial & 17.154 & Total Parcial & 14.828 \\
\hline
\end{tabular}

Fonte: Elaborado pelos autores

$\mathrm{Na}$ Tabela 3 observa-se o agendamento de atendimento médico para pacientes totalizando 31.982. A inclusão do paciente no agendamento ocorre após triagem clínica, onde são executados os procedimentos para fins de diagnóstico e tratamento do paciente após encaminhamento de outro profissional médico para a instituição.

4.2 A INFLUÊNCIA DA TECNOLOGIA DA INFORMAÇÃO "fatores técnicos e não técnicos"

A função da TI focada em melhoria de software ou operacionalização do uso do computador, em decorrência da crescente demanda por novos desenvolvimentos exige um constante aperfeiçoamento profissional para capacitação de pessoal altamente especializado na organização. Em um cenário ideal as habilidades técnicas são divididas dentro da própria área de TI, reorganizando a estrutura organizacional da TI e permeando melhor distribuir responsabilidades.

A maioria dos processos da instituição depende da TI: a informatização das rotinas; a utilização de estrutura em rede para interação com a interconexão global e local.

Da informatização das rotinas 
Revista de Administração Hospitalar, v.10, n.3, pp. 75-88, setembro/dezembro, 2013/ Ligia Adriane Thibe, Paulo Cesar Diniz De Araújo, Marcia Ribeiro Maduro, Luiz Augusto De Carvalho Francisco Soares, Luis Claudio De Jesus Silva

Doação e Distribuição de Sangue: todas as rotinas referentes à doação de sangue que incluem os exames liberação da doação e dos hemocomponentes, onde são armazenados e gerenciados os dados do doador e seus respectivos exames de sorologia: HIV, HCV, Sífilis, Chagas, Hepatite B e imunologia: pesquisa de anticorpos irregulares, tipagem sanguínea, fator $\mathrm{RH}$, fenotipagem;

Exames de Laboratório para pacientes: utiliza um sistema adquirido para otimização da rotina de laboratório como diferencial competitivo na execução de exames laboratoriais;

\section{Da utilização de estrutura em rede}

Sistemas Web: a instituição utiliza sistemas que estão na plataforma web, interagindo com base de dados externa, a exemplo o da área administrativa como o Sistema de Administração Financeira Integrada (AFI) ou área técnica como Gerenciador do Sistema Multicêntrico (GMS-NAT) do Ministério da Saúde;

Comunicação remota: a central de laboratório comunica-se com as subunidades (agências transfusionais) alocadas dentro de alguns hospitais e com a sede da Fundação de Apoio ao Hemoam (Fundação Sangue Nativo);

Sistemas em rede: a organização possui o Sistema de Acompanhamento à Doação de Sangue; Sistema de Cadastro Funcional; Sistema de Laboratório; Sistema de Prontuário Eletrônico; Sistema de Protocolo; Sistema de Planejamento.

\subsection{O PAPEL DA TI NO PROCESSO DECISÓRIO}

O setor de TI normalmente é um processo que funciona como apoio técnico da instituição; a constante mudança e inovação das atividades fins da organização com nível de complexidade crescente requer cada vez mais da capacidade técnica da TI. Dessa forma, o aperfeiçoamento e a qualificação profissional, oferecem à organização a oportunidade de minimizar os riscos gerados no uso da tecnologia.

A ação da Tecnologia da Informação e seus recursos, sem organização antecipada, não atinge seu principal objetivo de auxiliar a empresa em todos os seus processos e níveis de ação. O primeiro passo da tecnologia não é dispor de tecnologia, é vislumbrar a tecnologia de que se precisa e planejar como usá-la da melhor maneira. O software, o computador e seus periféricos são apenas importantes instrumentos de organização e não um fim em si mesmo. (Rezende, 2011).

Já para Castells, o valor agregado é gerado principalmente pela inovação, tanto no processo como de produtos. Assim, a questão é preciso um olhar diferenciado para os problemas não mensurados, ou seja, os riscos causados pelas falhas de TI são grandes, porém a equipe de TI precisa fortalecer este conhecimento. É preciso com clareza definir o que se precisa para atingir os objetivos. Para tal, o planejamento estratégico da TI deve preceder qualquer ação tomada para atingir os objetivos estratégicos dos serviços institucionais prestados. (Castells, 1999).

Diante do exposto, observa-se que para o setor de TI da instituição é imprescindível à atualização de novas tecnologias, o nivelamento de pessoal e o planejamento no gerenciamento dos recursos humanos realinhados; estabelecendo uma revisão, assegurando as habilidades; e a consciência de interação dos indivíduos, onde as atribuições correspondam às expectativas individuais e da instituição.

Das atividades desempenhadas pela TI para melhor controle precisam assumir um papel de transparência na organização; definir a implementação dos processos com base nas regras de responsabilidade vinculada aos processos de serviços da instituição. 
Revista de Administração Hospitalar, v.10, n.3, pp. 75-88, setembro/dezembro, 2013/ Ligia Adriane Thibe, Paulo Cesar Diniz De Araújo, Marcia Ribeiro Maduro, Luiz Augusto De Carvalho Francisco Soares, Luis Claudio De Jesus Silva

O monitoramento dos processos de TI é feito pelo armazenamento do atendimento aos usuários, totalizando uma média de 490 atendimentos/mês, das quais as informações são alocadas em uma base de dados de um sistema de intranet. Esses dados e o indicador de disponibilidade de sistema, conforme a Tabela 4, não é suficiente para mensurar e atuar como requisitos de controle para domínio e gerenciamento de desempenho da TI, e propor as melhorias que os serviços prestados pela TI requerem.

Do armazenamento dos dados da doação de sangue que geram a informação desde o ato da doação até a distribuição dos hemocomponentes ocorre via sistema, onde os dados são armazenados em uma base única de dados. No entanto, para fins de extração da informação para relatório gerencial não existe um framework para consolidação dos dados, e quando estas informações são necessárias a extração é realizada diretamente na base de dados pela equipe da TI, não estando disponível de imediato para o corpo gerencial, tornando inexistente o processo de controle e auditoria de Tecnologia da Informação.

Outros três pontos que precisam de freqüência na avaliação são: se os recursos investidos em TI, estão sendo suficiente para suportar os requisitos da instituição; se a entrega dos serviços e produtos de TI respeita os prazos acordados com os serviços; a satisfação do usuário dos serviços com relação aos serviços prestados pela equipe de TI.

Em suma, compreender as soluções a serem desenvolvidas ou adquiridas deve fazer parte da estratégia da organização e ser transparente para a gestão dos processos de TI. Nenhuma solução dada em tecnologia é eficiente se não for garantida a continuidade do uso e a manutenção nas mudanças com foco no objetivo da instituição.

A tecnologia da informação está presente nos principais atos da organização conforme será demonstrado a seguir através do mapeamento de processos:

Os Projetos desenvolvidos pela TI interna e concluídos são: Sistema de Acompanhamento à Doação de Sangue (SAD); Sistema de Cadastro Funcional (SCAF); Sistema de Acompanhamento de Documentos e Processos (Protocolo); Reestruturação de rede lógica; Firewall de bloqueio a acessos indesejados; Monitoramento da Base de Dados; Sistema de Acompanhamento de Suporte ao Usuário (Chamar); Suporte ao usuário.

Quanto as Tecnologias e Serviços Terceirizados, são utilizados por compreender a melhor solução e apresentar menores riscos, bem como são considerados diferenciadores não critico para a organização: Sistema de Laboratório de Pacientes (SoftLab); Sistema de Prontuário Eletrônico (Idoctor); Sistema de Planejamento (HEMOPLAN); Manutenção de ativos de rede; Estrutura de Backup de Dados; Sistema de Antivirus; Sistema de Monitoramento de Temperatura do Ambiente e Câmaras Frias; Sistema Gerenciador do Sistema Multicêntrico do Ministério da Saúde (GSM-NAT); Email Corporativo do Estado (Expresso); Serviço de Hospedagem de Site do Hemoam; Link de Internet (Metromao);

Os processos apresentados na Tabela 4 são desempenhados nas atividades do ciclo da doação de sangue e atendimento à paciente de forma informatizada, onde estão demonstrados as atividades prioritárias da instituição no que tange os "registros da doação e distribuição de sangue via sistema", o "registro de atendimento médico" e o "registro de exames de pacientes (SoftLab)" todos em relação a "Serviços de TI", "Indicadores da atividade" e "indicador da TI".

É bom ressaltar a importância da disponibilidade dos serviços de TI para o funcionamento dos processos demonstrados na Tabela 4. Essa ação busca o cumprimento das responsabilidades organizacionais relacionadas à gestão e monitoramento da tecnologia da informação, provendo a qualidade dos serviços prestados ao doador e ao paciente. Contudo, observa-se que não são todos os processos que possuem indicadores para dirimir problemas com indisponibilidade dos sistemas informatizados. 


\begin{tabular}{|c|c|c|c|}
\hline $\begin{array}{l}\text { Atividades Prioritárias } \\
\text { da Instituição }\end{array}$ & Serviços de TI & Indicador da atividade & Indicador da TI \\
\hline $\begin{array}{lclr}\text { Registro } & \text { da } & \text { doação } & \text { e } \\
\text { distribuição } & \text { de } & \text { sangue } & \text { via } \\
\text { sistema. } & & & \\
\end{array}$ & $\begin{array}{lr}\text { Sistema } & \text { de } \\
\text { Acompanhamento à } \\
\text { Doação de Sangue } \\
\text { (SAD). } \\
\text { Viabilidade de } \\
\text { infraestrutura } \\
\text { tecnológica. } \\
\begin{array}{l}\text { Banco de dados da } \\
\text { doação. }\end{array}\end{array}$ & $\begin{array}{l}\text { Dados estatísticos da doação } \\
\text { disponíveis. }\end{array}$ & $\begin{array}{ll}\text { Percentual } & \text { de } \\
\text { disponibilidade } & \text { do } \\
\text { sistema. } & \end{array}$ \\
\hline $\begin{array}{l}\text { Registro de Atendimento } \\
\text { Médico. }\end{array}$ & $\begin{array}{l}\text { Sistema de } \\
\text { Agendamento } \\
\text { Prontuário de Paciente } \\
\text { (Idoctor). } \\
\text { Banco de dados de } \\
\text { Sistema } \\
\text { Agendamento de } \\
\text { Prontuário de Paciente }\end{array}$ & $\begin{array}{l}\text { Atendimento de pacientes } \\
\text { agendados } \\
\begin{array}{l}\text { Dados do histórico de } \\
\text { pacientes. }\end{array}\end{array}$ & $\begin{array}{l}\text { Percentual } \\
\text { disponibilidade } \\
\text { sistema. } \\
\text { Não existe. }\end{array}$ \\
\hline $\begin{array}{l}\text { Registro de exames de } \\
\text { Pacientes (SoftLab). }\end{array}$ & $\begin{array}{ll}\begin{array}{l}\text { Sistema } \\
\text { Laboratório. }\end{array} & \text { de } \\
\text { Banco de dados do } \\
\text { Sistema } & \text { de } \\
\text { Laboratório } & \\
\end{array}$ & $\begin{array}{l}\text { Transação de exames de } \\
\text { pacientes } \\
\text { Dados estatísticos de exames } \\
\text { de pacientes. }\end{array}$ & $\begin{array}{ll}\begin{array}{l}\text { Percentual } \\
\text { disponibilidade }\end{array} & \text { do } \\
\text { sistema. } & \\
\text { Não existe. } & \end{array}$ \\
\hline
\end{tabular}

Fonte: Elaborado pelos autores

\section{CONSIDERAÇÕES FINAIS}

Alguns aspectos interessantes puderam ser observados, considerando pontos positivos e negativos, dificuldades e sucessos. Ao longo da transição entre a agência transfusional do Hospital Universitário Getúlio Vargas e a atual Fundação Hospitalar Hemoam o uso da Tecnologia da Informação têm aspectos inerentes a melhoria continua dos processos. E ainda que a ênfase da organização seja na área do ciclo do sangue, a aquisição de soluções tecnológicas tem permeado condições que despertam o interesse dos colaboradores da TI em compor o quadro da instituição.

Portanto, acreditamos que é possível se construir melhorias no desempenho das atividades técnicas de TI através de um diagnóstico de pessoal vinculado a área, ressaltando a formação e competência; levantando a necessidade de capacitação dos colaboradores e atribuindo responsabilidades. O envolvimento nas ações da TI é um fator fundamental que precisa ser trabalhado na organização; os processos prioritários precisam ser definidos democraticamente. O caminho em busca deste processo é o planejamento que pode ser construído pela constituição de um Comitê de TI, baseado no processo decisório participativo, alinhando as ações da TI com a estratégia da instituição.

Diante do exposto, a primeira etapa a ser vencida é a elaboração do diagnóstico da situação atual da TI e quais suas necessidades. Não de uma forma individual, mas através do Comitê de TI num processo dinâmico de planejamento ajustado as necessidades apresentadas, sendo estas detalhadas por nível de prioridade. Essa nova lógica, caracterizada por um novo paradigma na Tecnologia da Informação da organização, precisa promover recursos para 
Revista de Administração Hospitalar, v.10, n.3, pp. 75-88, setembro/dezembro, 2013/ Ligia Adriane Thibe, Paulo Cesar Diniz De Araújo, Marcia Ribeiro Maduro, Luiz Augusto De Carvalho Francisco Soares, Luis Claudio De Jesus Silva

reflexão das metas da TI que representem desafios possíveis de serem atingidos; melhorias no fluxo de atividades; interação e maior comprometimento dos colaboradores da TI com a organização; desmembramento da estrutura organizacional da TI; redefinição de tarefas; implantação de um modelo de Governança em TI para melhoria dos processos; agregação de talentos; novos projetos de desenvolvimento e implantação de uma ferramenta para gerenciamento e extração de dados. Além disso, para área de TI pesquisada percebeu-se que o mecanismo de avaliação de desempenho requer melhorias, e a compatibilidade das ações de TI alinhar com a estratégia da organização, esclarecendo assim gastos necessários para obtenção da eficiência nas atividades.

Em uma análise final, concluímos que é irrefutável a necessidade de se trabalhar com novas tecnologias, inovar continuamente os recursos e acompanhar as tendências tecnológicas, e ao mesmo tempo urge a necessidade da implantação de mecanismos para a melhoria dos processos e metas da Tecnologia da Informação. Afinal, investir ao longo do tempo para o aperfeiçoamento dos processos é importante, sobretudo a capacidade de gerir a informação é o caminho da prosperidade para a organização.

\section{REFERÊNCIAS}

ALBERTIN, Alberto Luiz e colaboração de MOURA, Rosa Maira de. Administração de Informática: funções e fatores críticos de sucesso. $4^{\mathrm{a}}$ ed. São Paulo: Atlas, 2002.

ANDRÉ, Marli E. D. A Pesquisa em Educação: Abordagens Qualitativas. São Paulo: EPU, 1986.

ARAÚJO, Paulo César Diniz; LIMA, Orlem Pinheiro; MADURO, Márcia Ribeiro; TERCEIRO NETO, Manoel Rodrigues. Guia Orientativo do Estágio Curricular Supervisionado do Curso de Administração. Manaus: UEA, 2010.

CASTELLS, Manuel. A Sociedade em Rede - a Era da Informação: Economia, Sociedade e Cultura. $6^{\text {a }}$ ed. São Paulo: Paz e Terra, 1999.

CHIAVENATO, Idalberto. Introdução à Teoria Geral da Administração: Uma Visão Abrangente da Moderna Administração das Organizações. 7.ed. ver. e atual. Rio de Janeiro: Elsevier, 2003 - $5^{\text {a }}$ Reimpressão.

COLEÇÃO PRÊMIO, Luís Eduardo Magalhães. A Revolução tecnológica, a Economia do conhecimento e a Democracia. Democracia: o Espaço da paz - Império e ditadura: a geopolítica da guerra. $6^{\text {a }}$ ed. Brasília: Instituto Tancredo Neves, 2004.

DRUCKER, Peter Ferdinand. O Gestor Eficaz. Rio de Janeiro: LTC, 2011.

MALVINO, Albert Paul. Microcomputadores e Microprocessadores. São Paulo: MacGrawHill do Brasil, 1985.

O'BRIEN, James A. Sistemas de Informação e as Decisões Gerenciais na Era da Internet. São Paulo: Saraiva, 2010.

PRAHALAD, C.K e HAMEL, Gary. Competindo pelo Futuro: estratégias inovadoras para obter o controle do seu setor e criar os mercados de amanhã. Rio de Janeiro: Elsevier, 2005 $12^{\mathrm{a}}$ Reimpressão.

REZENDE, Denis Alcides e ABREU, Aline França de. Tecnologia da Informação aplicada a Sistemas de Informação Empresariais: o papel estratégico da informação e dos sistemas de informação nas empresas. São Paulo: Atlas, 2011 - $8^{\text {a }}$ ed. 
Revista de Administração Hospitalar, v.10, n.3, pp. 75-88, setembro/dezembro, 2013/ Ligia Adriane Thibe, Paulo Cesar Diniz De Araújo, Marcia Ribeiro Maduro, Luiz Augusto De Carvalho Francisco Soares, Luis Claudio De Jesus Silva

SITES: Acesso em 20/11/2012: http://www.hemoam.org.br

VERGARA, Sylvia Constant. Projetos e Relatórios de Pesquisa em Administração. 13 ${ }^{\mathrm{a}}$ ed. São Paulo: Atlas, 2011. 